\title{
Jet energy scale uncertainty and resolution in the ATLAS experiment
}

\author{
Caterina Doglioni ${ }^{* *}$ \\ On behalf of the ATLAS collaboration \\ University of Oxford \\ E-mail: caterina.doglioniecern.ch
}

\begin{abstract}
About one year after the first proton proton collisions at a centre of mass energy of $\sqrt{s}=7 \mathrm{TeV}$ the ATLAS experiment has achieved a systematic uncertainty on the jet energy measurement down to $2.5 \%$ for jet transverse momenta from $20 \mathrm{GeV}$ to $2 \mathrm{TeV}$ in the pseudo-rapidity region up to $\eta=4.5$. The jet energy scale uncertainty is derived from in-situ single hadron response measurements along with systematic variations of the full Monte Carlo detector simulation. In addition, the transverse momentum balance between a central and a forward jet in events with only two jets at high transverse momentum is exploited. The uncertainty obtained is confirmed by direct in-situ measurements using the transverse momentum balance between a jet and a well measured reference, like the photon transverse momentum in photon-jet events or the total transverse momentum of the tracks matched to the jet. The jet response in the high energy regime can be validated using a system of well calibrated jets at low transverse momenta against a high-pt jet. The jet energy resolution can be determined in in-situ from the measurement of the transverse momentum balance of a system of two jets (transverse momentum asymmetry). The measurement is based on the direct transverse momentum balance and on the decomposition of the sum of the transverse jet momentum of the two jets along their bisector. Good agreement between data and Monte Carlo simulations is found. Sophisticated jet calibration schemes based on cell energy weighting or exploiting the internal jet structure are also presented. Such calibration schemes improve the jet resolution by $20-30 \%$ and in addition reduce the flavour dependence of the jet response.
\end{abstract}

The 2011 Europhysics Conference on High Energy Physics-HEP 2011,

July 21-27, 2011

Grenoble, Rhône-Alpes France

*Speaker.

${ }^{\dagger}$ Now at the Université de Genève 


\section{Introduction}

Hadronic jets are a fundamental ingredient for precision tests of QCD: understanding and measuring their performance is crucial in the LHC environment. This contribution presents selected results on the jet reconstruction performance in the ATLAS experiment obtained over the course of the 2010 and 2011 data taking, focusing on jet energy scale and resolution.

\section{Jet energy scale: calibration and uncertainty}

Jets are reconstructed in ATLAS with the anti- $k_{t}$ [1] algorithm with distance parameters $R=$ 0.4 and $R=0.6$. The EM+JES calibration [2] restores the hadronic jet energy scale (JES) using a data-derived offset pile-up subtraction [3] and employing $\left(\eta^{1}\right.$, E)-dependent calibration constants derived from Pythia [4] Monte Carlo simulations. The EM+JES scheme is used to calibrate jets with transverse momenta $p_{\mathrm{T}}^{\text {jet }}>20 \mathrm{GeV}$ and pseudorapidities up to $|\eta|<4.5$, and allows the JES uncertainty to be estimated directly.

The JES uncertainty is the dominant experimental uncertainty for numerous physics results at the LHC. A first estimate [5, 6] was based on information available before the first collisions and relied on the transverse momentum balance between a central and a forward jet for higher jet pseudorapidities as shown in Figure 1 (a). The calibration and the JES uncertainty were updated including the full $35 \mathrm{pb}^{-1}$ collected in 2010 [2]. Data-derived techniques were employed, including the calibration of the absolute calorimeter electromagnetic energy scale from $Z$-boson decays in the electron channel $(Z \rightarrow e e)$ [7] and precise in-situ measurements of the single hadron response [8]. This leads to a significant reduction of the JES uncertainty in the central detector region, as it can be seen in Figure 1 (b). The smallest JES uncertainty of less than $2.5 \%$ is found in the central calorimeter region $(|\eta|<0.8)$ for jets with $60 \mathrm{GeV} \leq p_{\mathrm{T}}^{\text {jet }}<800 \mathrm{GeV}$. The JES uncertainty is the

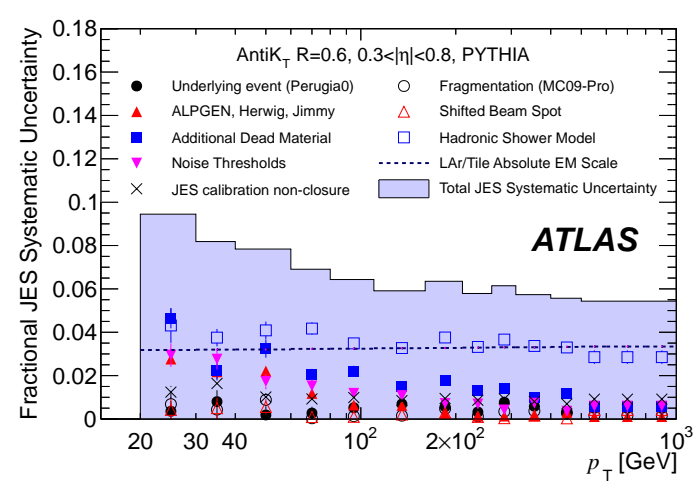

(a)

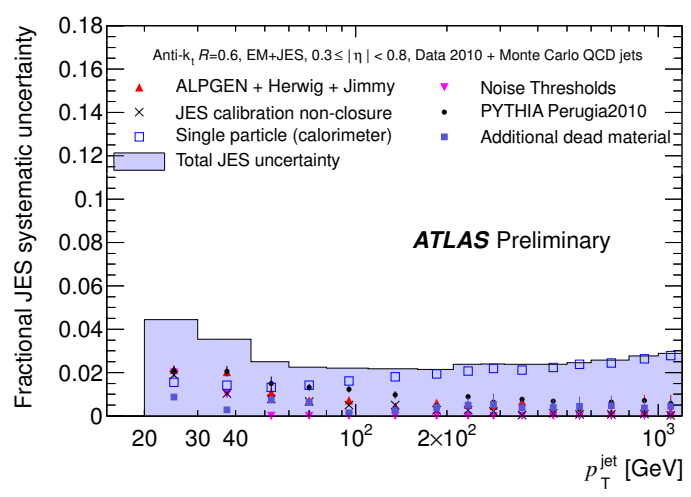

(b)

Figure 1: Figure(a): JES uncertainty for the first ATLAS data (July 2010) [6]. Figure (b): JES uncertainty after analysis of first collision data (March 2011) [2].

\footnotetext{
${ }^{1}$ The ATLAS coordinate system is a right-handed system with the $x$-axis pointing to the centre of the LHC ring, the $z$-axis following the beam direction and the $y$-axis pointing upwards. The pseudorapidity $\eta$ is related to the polar angle $\theta$ as $\eta=-\ln \tan \frac{\theta}{2}$.
} 
largest for low- $p_{\mathrm{T}}\left(20 \mathrm{GeV} \leq p_{\mathrm{T}}^{\text {jet }}<30 \mathrm{GeV}\right)$ jets in the most forward region $3.2 \leq|\eta|<4.5$ where it amounts to $14 \%$.

The number of interactions in the same bunch crossing (in-time pile-up) and the amount of overlapping signal from other collisions in nearby bunch crossings (out-of-time pile-up) increased significantly from 2010 to 2011. In 2010, the remaining pile-up uncertainty (added in quadrature to the baseline 2010 uncertainty) is less than $1.5 \%$ per additional interaction for jets with $p_{\mathrm{T}}^{\text {jet }}>$ $50 \mathrm{GeV}$ and decreases with $p_{\mathrm{T}}$, thanks to the removal of additional energy due to in-time pile-up in the calibration scheme. In early 2011 measurements this correction was not applied. Instead, it was used to estimate the pile-up uncertainty amounting to $5 \%$ (7\%) for jets of $20<p_{\mathrm{T}}^{\text {jet }}<50 \mathrm{GeV}$ in the range $0 \leq|\eta|<2.8(2.8 \leq|\eta|<4.4)$ and to $2 \%(3 \%)$ for jets of $50<p_{\mathrm{T}}^{\text {jet }}<100 \mathrm{GeV}$ in the range $0 \leq|\eta|<2.8(2.8 \leq|\eta|<4.4)$.

\section{Jet energy scale validation with in-situ techniques}

Jet reconstruction, and in particular the Monte Carlo based jet energy scale calibration, can be validated using in-situ techniques that compare data and simulation. In-situ techniques use well measured benchmark objects that balance the jet to evaluate the absolute jet energy scale. The calibration of low $p_{\mathrm{T}}$ jets is validated exploiting the $p_{\mathrm{T}}$ balance of the jet with a well-measured photon in the direct balance and missing- $E_{\mathrm{T}}$ projection fraction (MPF) methods [9]. The dielectron invariant mass in $Z \rightarrow e e$ events can be used to check the scale of recoiling jets with the statistics available in 2011. Jets made of inner detector tracks can be compared to the corresponding jet measured from calorimeter energy deposits [10]. The balance between the leading jet and the recoil system (multi-jet balance) is used as a probe for high- $p_{\mathrm{T}}$ jets [11]. The 2010 data/Monte Carlo ratio can be found in Figure 2 (a).

Due to the increased pile-up in 2011 data, the in-situ validation of the jet energy scale is particularly relevant. As an example, the data/MC comparison for the multi-jet balance of Figure 2 (b) shows that a good understanding of jets up to $1.4 \mathrm{TeV}$ is reached with the current calibration and that the uncertainties are in agreement with those obtained in the 2010 data.

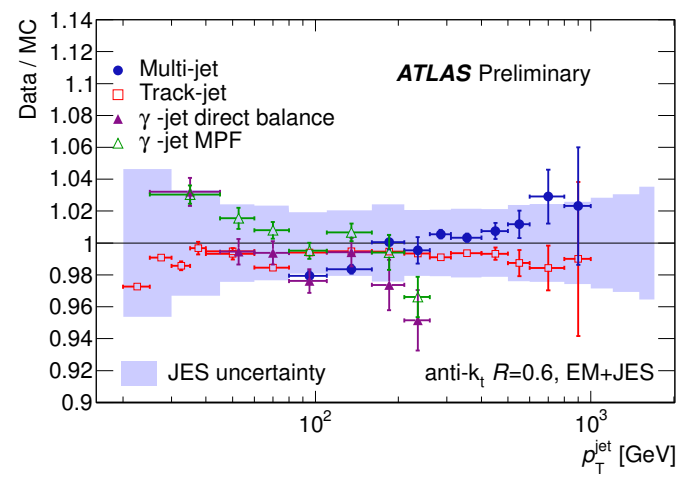

(a)

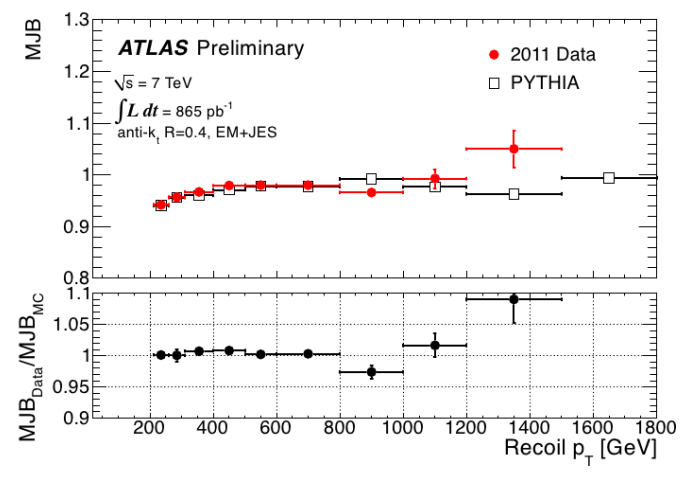

(b)

Figure 2: Figure (a): In-situ balance for EM+JES calibrated jets in 2010 data and Monte Carlo [2]. Figure (b): Multijet balance in 2011 data and Monte Carlo (ratio data/Monte Carlo shown in the lower panel) [12]. 


\section{Jet energy resolution}

ATLAS employs two independent techniques to measure the jet energy resolution using calorimeter observables: the dijet balance method and the bisector method [13]. These rely on different assumptions and have different systematic uncertainties, providing separate cross-checks of the data/Monte Carlo agreement of the jet energy resolution. The dijet balance method relies on energy conservation in the transverse plane in dijet events. Its total systematic uncertainty has been evaluated in 2010 and it amounts to $5-8 \%$ in the $p_{\mathrm{T}}^{\text {jet }}$ range of $20-500 \mathrm{GeV}$. The bisector method uses the decomposition of the vector sum of the two leading jet momenta to estimate the jet transverse momentum resolution, with an uncertainty of 3-4\% in the $p_{\mathrm{T}}^{\text {jet }}$ range of $20-500 \mathrm{GeV}$ in 2010.

Refined calibration techniques [15] are undergoing commissioning: these make use of hadronic shower properties thus reducing the jet energy fluctuations and the sensitivity of the jet response to the jet flavour. The Global Sequential Calibration (GS) exploits the properties of energy depositions in calorimeter layers, the Global Cell Weighting (GCW) uses the cell energy density to weight cells within the jet and the Local Cluster Weighting (LCW) employs factorized corrections derived from cluster properties in simulations of single pions. The comparison of the jet resolution for the various calibration schemes, evaluated using the bisector method, is shown in Figure 3 (a). The data/Monte Carlo agreement for most calibrations and $p_{\mathrm{T}}$ bins is within uncertainties, and the use of refined calibration techniques brings an improvement of up to $30 \%$ in the jet energy resolution when compared to the EM+JES scheme. Figure 3 (a) compares the jet energy resolution for EM+JES calibrated jets in 2010 and 2011: the agreement is within $10 \%$ for $p_{\mathrm{T}}^{\text {jet }}>40 \mathrm{GeV}$.

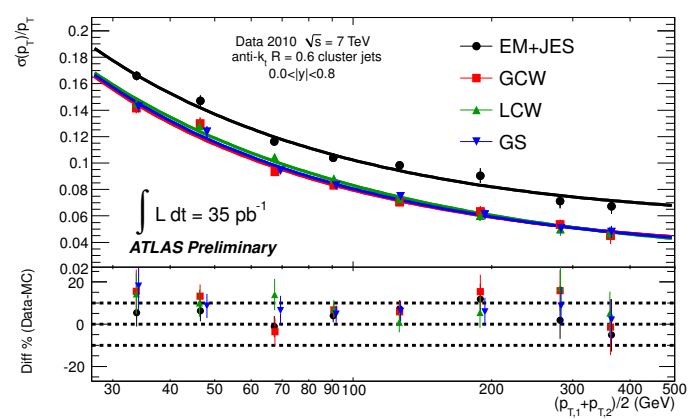

(a)

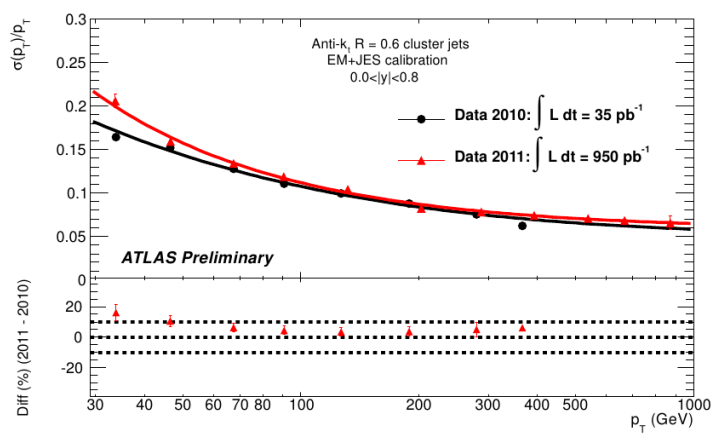

(b)

Figure 3: Fractional jet energy resolution as a function of the average jet transverse momentum measured with the bisector in-situ technique, with fit to data overlaid. Figure (a): Comparison of various calibration schemes, with relative difference between Monte Carlo and data results in the lower plot. Figure (b): EM+JES calibration, comparison of 2011 and 2010 data results, with relative difference between 2011 and 2010 results in the lower plot. Figures from Reference [14].

\section{Conclusions and outlook}

A good understanding of the jet energy scale and resolution in the ATLAS experiment has been reached in 2010 and confirmed in 2011 using in-situ techniques. It is expected that the jet energy resolution will benefit from refined calibration techniques undergoing commissioning. 


\section{References}

[1] M. Cacciari, G. P. Salam, G. Soyez, The anti- $k_{t}$ jet clustering algorithm, JHEP 04 (2008) 063 [hep-ph/0802.1189]; Fastjet, http://fastjet.fr/

[2] The ATLAS Collaboration, Jet energy scale and its systematic uncertainty in proton-proton collisions at $\sqrt{s}=7 \mathrm{TeV}$ in ATLAS 2010 data, ATLAS-CONF-2010-032, http://cdsweb.cern.ch/record/1337782

[3] The ATLAS Collaboration, In-situ jet energy scale and jet shape corrections for multiple interactions in the first ATLAS data at the LHC, ATLAS-CONF-2011-030, http://cdsweb.cern.ch/record/1337780

[4] T. Sjostrand, S. Mrenna, P. Z. Skands, PYTHIA 6.4 Physics and Manual, JHEP 05 (2006) 026 [hep-ph/0603175]

[5] The ATLAS Collaboration, Jet energy scale and its systematic uncertainty for jets produced in proton-proton collisions at $\sqrt{s}=7 \mathrm{TeV}$ and measured with the ATLAS detector, ATLAS-CONF-2010-056, http://cdsweb.cern.ch/record/1281329

[6] The ATLAS Collaboration, Measurement of inclusive jet and dijet cross sections in proton-proton collisions at 7 TeV centre-of-mass energy with the ATLAS detector, Eur. Phys. J. C71 (2011) 1512 [hep-ex/1109.5908]

[7] The ATLAS Collaboration, Electron performance measurements with the ATLAS detector using the 2010 LHC proton-proton collision data, [hep-ex/1110 . 3174]

[8] The ATLAS Collaboration, ATLAS calorimeter response to single isolated hadrons and estimation of the calorimeter jet scale uncertainty, ATLAS-CONF-2011-028, http://cdsweb.cern.ch/record/1337075

[9] The ATLAS Collaboration, Determination of the jet energy measurement uncertainty using photon-jet events in proton-proton collisions at $\sqrt{s}=7 \mathrm{TeV}$, ATLAS-CONF-2011-031, http://cdsweb.cern.ch/record/1337781

[10] The ATLAS Collaboration, Determination of the ATLAS jet energy measurement uncertainties using tracks in proton proton collisions at $\sqrt{s}=7 \mathrm{TeV}$, ATLAS-CONF-2011-067, http://cdsweb.cern.ch/record/1349308

[11] The ATLAS Collaboration, Probing the jet energy measurement at the TeV-scale using the multi-jet balance technique in proton-proton collisions at $\sqrt{s}=7$ TeV, ATLAS-CONF-2011-029, http://cdsweb.cern.ch/record/1337076

[12] The ATLAS Collaboration, Jet/MissingEt preliminary results on multi-jet balance from 2011 data, https://twiki.cern.ch/twiki/bin/view/AtlasPublic/JetEtmissApproved2011MultiJetBalance

[13] The ATLAS Collaboration, Jet energy resolution and selection efficiency relative to track jets from in-situ techniques with the ATLAS Detector Using Proton-Proton Collisions at a Center of Mass Energy $\sqrt{s}=7 \mathrm{TeV}$, ATL-COM-PHYS-2010-054, http://cdsweb.cern.ch/record/1281311

[14] The ATLAS Collaboration, Jet/MissingEt preliminary results on jet resolution from 2011 data, https://twiki.cern.ch/twiki/bin/view/AtlasPublic/JetEtmissApproved2011JetResolution

[15] The ATLAS Collaboration, Properties of Jets and Inputs to Jet Reconstruction and Calibration with the ATLAS Detector Using Proton-Proton Collisions at $\sqrt{s}=7 \mathrm{TeV}$, ATLAS-CONF-2011-053, http://cdsweb.cern.ch/record/1281310 